\title{
Evaluating the Association of Chronic Periodontitis with Quality and Duration of Sleep in North Indian Population Using Pittsburgh Sleep Quality Index - A Case Control Study
}

\section{Varsha Kanjani ${ }^{1 *}$, Deepak Kanjani ${ }^{2}$, Venkata Sai Teja Mungara ${ }^{3}$, Amba Shree Bharadwaj ${ }^{4}$, Chitra, Chandrashekhar Maggidwar ${ }^{5}$}

${ }^{1}$ Department of Oral Medicine and Radiology, Vyas Dental College and Hospital, Jodhpur, Rajasthan, India

${ }^{2}$ Medical Officer, Fidusar Government Hospital, Jodhpur, Rajasthan, India

${ }^{3}$ Department of Prosthodontics, Crown and Bridge Including Implantology, C.K.S

Teja Institute of Dental Sciences and Research, Tirupati, Andhra Pradesh, India

${ }^{4}$ Department of Oral Medicine and Radiology, Manipal College of Dental Sciences, Manipal, India

${ }^{5}$ Department of Oral Medicine and Radiology, Nanded Rural Dental College and Research Center, Nanded, India

*Corresponding Author: Varsha Kanjani, Department of Oral Medicine and Radiology, Vyas Dental College and Hospital, Jodhpur, Rajasthan, India.
Received: December 02, 2021

Published: January 21, 2022

(C) All rights are reserved by Varsha Kanjani., et al.

\begin{abstract}
Objectives: Sleep is a biological phenomenon, greatly affecting psychological and physical health of an individual. The mechanism behind the association between chronic periodontitis and deprived sleep is still not clear. Deprived or disturbed sleep has said to be associated with increased levels of inflammatory markers, thereby leading to development of chronic periodontal diseases. The present study was conducted to evaluate the association of chronic periodontitis with quality and duration of sleep in North Indian population.

Materials and Methods: The present case control study involving total of 340 individuals, taken into cases (individuals with chronic periodontitis) and controls (individuals without chronic periodontitis. The diagnosis was based on the Center of Disease Control and Prevention, described by Eka., et al. (2012). A structured performa was included containing demographic data and to determine the quality and duration of sleep, a validated Pittsburgh Sleep Quality Index was assessed. The data was analyzed using SPSS version 21.0 .

Results: On clinical examination, mean pocket depth and clinical attachment level in individuals with periodontitis were $4.09 \pm 0.48$ $\mathrm{mm}$ and $5.15 \pm 0.94 \mathrm{~mm}$ respectively. The present study stated poor quality of sleep-in individuals with periodontitis with $69.41 \%$ and the individuals with more than 7 hours of sleep were at more risk in developing periodontitis.

Conclusion: The poor sleep quality and longer sleep duration is significantly associated with chronic periodontitis. Age is another significant variable while other factors such as gender, educational qualification and socioeconomic status was insignificant.
\end{abstract}

Keywords: Chronic Periodontitis; Pittsburgh Sleep Quality Index; Sleep Duration; Sleep Quality

Citation: Varsha Kanjani., et al. "Evaluating the Association of Chronic Periodontitis with Quality and Duration of Sleep in North Indian Population Using Pittsburgh Sleep Quality Index - A Case Control Study". Acta Scientific Dental Sciences 6.2 (2022): 82-87. 
Evaluating the Association of Chronic Periodontitis with Quality and Duration of Sleep in North Indian Population Using Pittsburgh Sleep Quality Index - A Case Control Study

\section{Abbreviation}

PSQI: Pittsburgh Sleep Quality Index; PD: Periodontal Depth; CAL: Clinical Attachment Loss; SPSS: Statistical Package of Social Science

\section{Introduction}

Sleep is a complex biological phenomenon, critically associated with psychological and physical health. Periodontitis is multifactorial inflammatory disease-causing periodontal destruction and thus leading to periodontal pocket, bone loss and gradually tooth mobility $[1,2]$. The mechanism that sleeps is associated with systemic inflammation is not clear but few studies in literature stated elevated levels of inflammatory markers and common risk factors such as gender, age, habits like smoking, alcohol, obesity etc. are associated with disturbed sleep and chronic periodontitis [3,4].

Therefore, the present study was conducted to assess the association of chronic periodontitis with quality and duration of sleep in North Indian population.

\section{Materials and Methods}

The present case control study was conducted among 340 individuals from April to December 2018 in a Diagnostic setup, Jodhpur, Rajasthan. The study was approved by Government Hospital, Jodhpur, Rajasthan. All the individuals enrolled were informed about the study and written consent was obtained. The enrolled individuals were divided into healthy individuals (controls) and individuals with periodontitis (cases). The individuals with systemic diseases (such as HIV, hypertensive, diabetes mellitus, etc.), pregnant or lactating females or individuals who are edentulous or have taken any periodontal treatment in last 6 months were excluded from the study. The cases between 25-45 years of age, having at least 16 natural teeth, without any coexisting systemic illness were enrolled in the study [5].

A structured performa was given to each participant including demographic data (name, age gender, educational qualification and socioeconomic status) and Pittsburgh Sleep Quality Index (PSQI). PSQI is valid, effective and standardized questionnaire for determining the quality and pattern of sleep. The present effective instrument can be used for both adult and old age as stated in literature [6,7]. It contains 19 items, grouped into 7 domains score on the scale of 0 to 3 . Therefore, the complete score ranges from 0 to 21 where lesser score indicates good quality of sleep and as scores increases, the quality of sleep diminishes. The score 5 or more was considered as poor sleep. Incomplete performa's were excluded from the study.

The periodontal status of all the teeth present (excluding third molars) was assessed by single examiner, using William's graduated probe. The clinical examination involved pocket depth (PD) and clinical attachment loss (CAL) of six surfaces of all teeth present i.e., mesiolingual, midlingual, distolingual, mesiobuccal midbuccal and distobuccal surfaces. According to Center of disease control and prevention [8], the cases were diagnosed as chronic periodontitis, as narrated by Eke PI., et al. (2012) into mild, moderate and severe. The individuals, who could not meet the criteria, were considered as controls. In the present research, number of cases and controls were taken in ratio of 1:1.

The obtained data was analyzed using SPSS version 21.0. The descriptive data obtained in terms of mean, standard deviation and percentage while $t$-test were used for determining significance of pocket depth (PD) and clinical attachment loss (CAL). The logistic regression analysis was used to assess the relationship between univariate and multivariate data. The $\mathrm{p}$ value less than 0.05 were considered as significant.

\section{Results}

The mean age of cases (individuals with periodontitis) and controls (individuals without periodontitis) was $43.36 \pm 7.47$ and $39.62 \pm 11.82$ years. In the present study, $54.70 \%$ and $47.64 \%$ were males in cases and controls respectively. The quality of sleep was poor in cases than controls with percentage of 69.41 and 35.88 respectively while good quality sleep was seen in individuals without periodontitis. The individuals with more than 7 hours of sleep were at more risk in developing periodontitis than individuals who sleep less with $67.64 \%$ and $32.53 \%$ respectively with odd's ratio = $1.74 \%$ and Confidence interval $=1.17-3.62$. Table 1 summarizes the demographic data and quality of sleep in cases and controls.

The mean PSQI score was $1.04 \pm 0.73$ and $8.46 \pm 1.05$ in case of healthy and chronic periodontitis individuals respectively with intergroup statistical significance of 0.001 ( $p$ value).

On clinical examination, mean PD was $4.09 \pm 0.48 \mathrm{~mm}$ and1.29 $\pm 0.59 \mathrm{~mm}$ in cases and controls respectively. The CAL in cases was 
Evaluating the Association of Chronic Periodontitis with Quality and Duration of Sleep in North Indian Population Using Pittsburgh Sleep Quality Index - A Case Control Study

\begin{tabular}{|l|c|c|}
\hline Parameters & \multicolumn{1}{|c|}{$\begin{array}{c}\text { Cases } \\
\text { (With Periodontitis) }\end{array}$} & $\begin{array}{c}\text { Controls } \\
\text { (Without } \\
\text { Periodontitis) }\end{array}$ \\
\hline Age & $43.36 \pm 7.47$ & $39.62 \pm 11.82$ \\
\hline Gender & $93(54.70 \%)$ & $81(47.64 \%)$ \\
\hline Male & $77(45.29 \%)$ & $89(52.35 \%)$ \\
\hline Female & $37(21.76 \%)$ & $26(15.29 \%)$ \\
\hline Education & $69(40.58 \%)$ & $97(57 \%)$ \\
\hline $\begin{array}{l}\text { Higher } \\
\text { secondary }\end{array}$ & $64(37.64 \%)$ & $47(27.64 \%)$ \\
\hline Graduate & $115(67.64 \%)$ & $81(47.64 \%)$ \\
\hline Postgraduate & $55(32.35 \%)$ & $89(52.35 \%)$ \\
\hline $\begin{array}{l}\text { Quality of } \\
\text { sleep }\end{array}$ & $52(30.58 \%)$ & $109(64.11 \%)$ \\
\hline Good & $118(69.41 \%)$ & $61(35.88 \%)$ \\
\hline Poor & & \\
\hline $\begin{array}{l}\text { Duration of } \\
\text { sleep }\end{array}$ & & \\
\hline$>7$ hours & & \\
\hline$<7$ hours & & \\
\hline
\end{tabular}

$5.15 \pm 0.94 \mathrm{~mm}$ while in controls was $0.01 \pm 0.62 \mathrm{~mm}$. Both PD and CAD were statistically significant with $\mathrm{p}$ value of 0.001 (Table 2 and Figure 2). The clinical parameters were in positive association with PSQI score. The logistic regression analysis stated strong association of chronic periodontitis with quality of sleep (Odd's ratio $=1.89 \%$, Confidence interval $=1.02-3.39$ ). The present study stated highly significant association with age ( $\mathrm{p}$ value $=0.003$ ) but weak association of gender, education or socioeconomic status with chronic periodontitis.

\begin{tabular}{|l|c|c|c|}
\hline \multirow{2}{*}{ Parameters } & \multicolumn{2}{|c|}{ Mean \pm SD } & \multirow{2}{*}{} \\
\cline { 2 - 3 } & $\begin{array}{c}\text { Cases } \\
\text { (With } \\
\text { Periodontitis) }\end{array}$ & $\begin{array}{c}\text { Controls } \\
\text { (Without } \\
\text { Periodontitis) }\end{array}$ & \\
\hline $\mathrm{PD}^{\dagger}(\mathrm{mm})$ & $4.09 \pm 0.48$ & $1.29 \pm 0.59$ & $0.001^{*}$ \\
\hline $\mathrm{CAL}^{\ddagger}(\mathrm{mm})$ & $5.15 \pm 0.94$ & $0.01 \pm 0.62$ & $0.001^{*}$ \\
\hline $\mathrm{PSQI}^{\S} \mathrm{score}$ & $8.46 \pm 1.05$ & $1.04 \pm 0.73$ & $0.001^{*}$ \\
\hline
\end{tabular}

Table 2: Clinical parameters measured in cases and controls.

${ }^{*}$ p value more than 0.05 was considered as significant

$$
\begin{aligned}
& \text { †Periodontal depth } \\
& { }^{\ddagger} \text { Clinical attachment loss }
\end{aligned}
$$

§Pittsburgh Sleep Quality index.

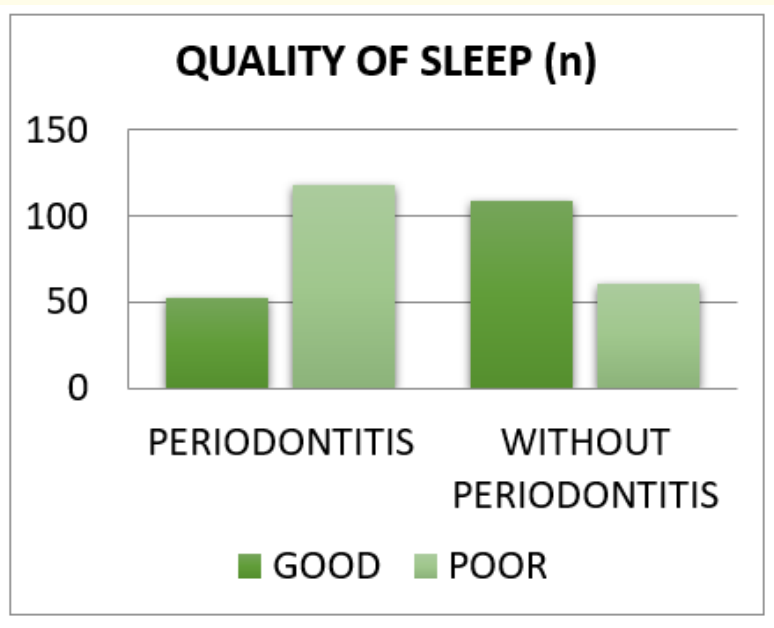

Figure 1: Quality of life in individuals with (cases) and without (controls) periodontitis.

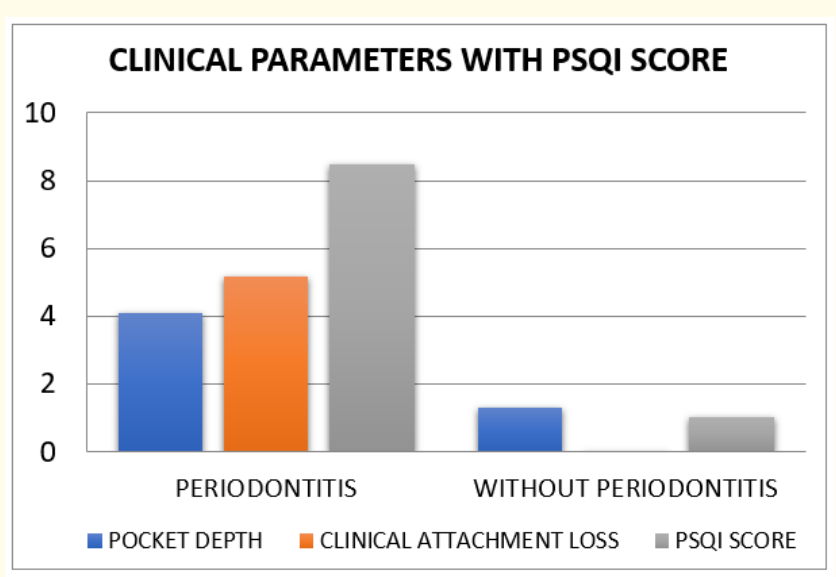

Figure 2: Clinical Parameters and PSQI score measured in individuals with (cases) and without (controls) periodontitis.

Citation: Varsha Kanjani., et al. "Evaluating the Association of Chronic Periodontitis with Quality and Duration of Sleep in North Indian Population Using Pittsburgh Sleep Quality Index - A Case Control Study". Acta Scientific Dental Sciences 6.2 (2022): 82-87. 
Evaluating the Association of Chronic Periodontitis with Quality and Duration of Sleep in North Indian Population Using Pittsburgh Sleep Quality Index - A Case Control Study

\section{Discussion}

Chronic periodontitis is multifactorial inflammatory disease, which mediates the microbial etiologic host responses thereby regulates inflammatory events. The microbial inflammatory mediators may enter the blood stream through periodontal tissues and leads to systemic inflammation in human body. These inflammatory mediators in systemic circulation, dysregulation of immune pathway and adaptive immunity are the major factors leading to chronic inflammation. The various systemic conditions associated with chronic periodontal diseases as stated in literature are cardiac disease, chronic kidney diseases, cognitive impairment, diabetes, respiratory diseases, rheumatoid arthritis, obesity, metabolic diseases, etc. $[9,10]$.

Sleep is a complex biological phenomenon, essential for daily human functioning and activities. The changes in lifestyle among today's generation have led to disturbances in sleep cycle thereby, inducing inflammatory reactions in body. Epidemiologic studies $[11,12]$ stated in literature have concluded that deprived or disturbed sleep adversely affect physical and mental health of an individual, in turn reducing immunity with increased state of inflammatory markers, causing systemic inflammatory events in human body [13]. The disturbances in sleep and wake cycles causes activation of hypothalamic pituitary adrenal axis and the sympathetic nervous system, thereby suppression the antiviral response, simultaneously stimulating pro-inflammatory genes such as Interleukin 4 and 5 . These can be possible mechanisms behind deprived or disturbed sleep-in individuals with chronic inflammation [14]. Thus; the primary objective of the present study was to evaluate the association of chronic periodontitis with quality and duration of sleep.

Various methods for assessing the quality, quantity and duration of sleep are used in literature such as clinical interviews, maintaining diaries or variety of questionnaires. PSQI used in present study, has reliability coefficient of 0.83 for its seven components with $89.6 \%$ sensitivity and $86.5 \%$ specificity [15]. The reliability of the above data was supported by Buysse DJ., et al. (1989) and Manzar MD., et al. (2016) [6,7]. The present study used the PSQI to assess the quality of sleep stated that poor sleep quality was seen in $118 / 170$ (69.41\%) individuals with chronic periodontitis while good sleep quality was observed in 109/170 (64.11\%) healthy individuals. The present study was in consonance with the studies published by Grover V., et al. (2015) [14] stating that PSQI score was higher in individuals with chronic periodontitis as compared to healthy individuals. The authors further concluded that the severities of periodontal diseases are proportional to the deprived or disturbed sleep pattern. Another study by Singh VP., et al. (2019) [15] stated the correlation of quality of sleep with periodontitis in Malaysian populations using PSQI index concluding significant association of poor sleep quality with chronic periodontitis in 56.75 $\%$ individuals.

Another study by Singla N (2016) [16] assessed the impact of variable lifestyle on periodontal health. The authors stated deprived or disturbed sleep as one of the significant cause, leading to progression of the periodontal diseases in Udupi population, Karnataka. The individuals who sleep for 7 to 8 hours / day exhibit lesser chances to develop periodontal diseases as compared to individuals who sleep 6 hours or lesser. Therefore, authors concluded the shorter sleep duration greatly affect the body's immunity, leading to periodontal diseases. Similar study conducted by Romandini M (2017) concluded independent and direct relationship between duration of sleep and chronic periodontitis among Korean population. The authors stated with every increased hour of sleep, the risk of periodontitis increases by $17 \%$ with bidirectional relationship [17].

The Korea National Health and Nutrition Examination survey conducted on 3292 women, stated the higher risk of periodontitis in individuals with 7 hours or more sleep duration than those with less than 7 hours (crude OR) by 1.37 times (95\% confidence interval, 1.13 1.65). The authors concluded the strong relation between risk of chronic periodontitis and duration of sleep among Korean women. They further emphasize on implementing a comprehensive health program, in association with oral hygiene awareness practices to improve the sleeping habits and reducing the risk of periodontitis in Korean women [18].

Another study published by Wiener RC (2016) [19]. was in disagreement with the present study stating no association between 7 hours duration of sleep and chronic periodontitis along with that author has used multiple examiners for clinical examination of participants and large data was obtained from the national survey conducted in $2009-2012$. While in the present study, there is strong association between sleep duration and chronic periodontitis, and clinical examination was performed by single qualified examiner. 
Evaluating the Association of Chronic Periodontitis with Quality and Duration of Sleep in North Indian Population Using Pittsburgh Sleep Quality Index - A Case Control Study

As chronic periodontal diseases are associated with various risk factors such as age, gender, educational qualification and socioeconomic status, were also assessed. Age is highly associated with the periodontal status of the individual. We included individuals between 25 -45 years of age but we could not match the age of cases with controls as most of the individuals with periodontitis are of higher age range which is in accordance with another study [15]. The present study stated highly significant association with age ( $\mathrm{p}$ value $=0.003$ ) similar to the other studies $[16,19]$ but weak association of gender, education or socioeconomic status with chronic periodontitis. In another study, gender is not significantly associated with risk of periodontitis [15]. A systemic review [20] and population-based study [21] stated that the severity of periodontal diseases are influenced by low educational qualification and socioeconomic status but in the present study, weak association was found which was in accordance with other reported studies [15].

As chronic periodontal diseases have multifactorial etiology, the present case control study has included various risk factors along with clinical parameters to fortify the present findings but can also be influenced by unknown possible cofactors which may influence the quality of sleep and chronic periodontitis.

\section{Conclusion}

The present case control study has shown significant association of chronic periodontitis with quality and duration of sleep. The poor sleep quality and longer sleep duration is significantly associated with chronic periodontitis. Age is another significant variable while other factors such as gender, educational qualification and socioeconomic status were insignificant. Therefore, we further recommend investigating the role of sleep with chronic periodontal diseases.

\section{Acknowledgement}

Nil.

\section{Conflict of Interest}

Nil.

\section{Financial Source/Support}

Nil.

\section{Current Knowledge}

The elevated levels of inflammatory markers in disturbed or deprived sleep, along with common risk factors, leads to its correlation with oral and periodontal diseases.

\section{Study Impact}

The chronic periodontal diseases present a high impact on the quality and duration of sleep in a North Indian population. Thus, poor sleep quality and longer sleep duration affects the oral health related quality of life.

Evaluating the association of chronic periodontitis with quality and duration of sleep in North Indian population using Pittsburgh Sleep Quality index - a case control study.

\section{Bibliography}

1. Keller JJ., et al. "Association between obstructive sleep apnoea and chronic periodontitis: a population-based study". Journal of Clinical Periodontology 40.2 (2013): 111-117.

2. Linden GJ., et al. "Periodontal systemic associations: Review of the evidence". Journal of Clinical Periodontology 84.4s (2013): 8-19.

3. Nizam N., et al. "Salivary cytokines and the association between obstructive sleep apnea syndrome and periodontal disease". Journal of Periodontology 85.7 (2014): 251-258.

4. Al-Jewair TS., et al. "Periodontitis and obstructive sleep apnea's bidirectional relationship: a systematic review and meta-analysis". Sleep Breath 19.4 (2015): 1111-1120.

5. Loke W., et al. "Investigating the association betweenobstructive sleep apnea and periodontitis". Journal of Periodontology 86.2 (2015): 232-243.

6. Buysse DJ., et al. "The pittsburgh sleep quality index: A new instrument for psychiatric practice and research". Psychiatry Research 28.2 (1989): 193-213.

7. Manzar MD., et al. "Dimensionality of the pittsburgh sleep quality index in the collegiate young adults". SpringerPlus 5.1 (2016): 1550

8. Eke PI., et al. "Advances in surveillance of periodontitis: The centers for disease control and prevention periodontal disease surveillance project". Journal of Periodontology 83.11 (2012): 1337-1342.

9. Cekici A., et al. "Inflammatory and immune pathways in the pathogenesis of periodontal disease". Periodontology 2000 64.1 (2014): 57-80.

10. Linden GJ., et al. "Periodontal systemic associations: Review of the evidence". Journal of Clinical Periodontology 40.14 (2013): S8-19. 
11. Singh M., et al. "The association between obesity and short sleep duration: A population-based study". Journal of Clinical Sleep Medicine 1 (2005): 357-363.

12. Parish JM and Somers VK. "Obstructive sleep apnea and cardiovascular disease". Mayo Clinic Proceedings 79.8 (2004): 1036-1046.

13. Irwin MR., et al. "Sleep deprivation and activation of morning levels of cellular and genomic markers of inflammation". Archives of Internal Medicine 166.16 (2006): 1756-1762.

14. Grover V., et al. "Exploring association between sleep deprivation and chronic periodontitis: A pilot study". Journal of Indian Society of Periodontology 19.3 (2015): 304-307.

15. Singh VP., et al. "Association between quality of sleep and chronic periodontitis: A case-control study in Malaysian population". Journal of Dental Research 16 (2019): 29-35.

16. Singla N., et al. "The impact of lifestyles on the periodontal health of adults in Udupi district: A cross sectional study". Journal of Indian Society of Periodontology 20.3 (2016): 330-335.

17. Romandini M., et al. "The association between periodontitis and sleep duration". Journal of Clinical Periodontology 44 (2017): 490-501.

18. Kyung-Y D and Eun-Sun L. "Relationship between Sleep Duration and Periodontitis in Korean Adult Women: Data from Korea National Health and Nutrition Examination Survey 2014". Journal of Dental Hygiene Science 17.4 (2017): 298-305.

19. Wiener RC. "Relationship of routine inadequate sleep duration and periodontitis in a nationally representative sample". Sleep disorder 2016 (2016): 9158195.

20. Boillot A., et al. "Education as a predictor of chronic periodontitis: A systematic review with meta-analysis population-based studies". PLoS One 6.7 (2011): e21508.

21. Buchwald S., et al. "Tooth loss and periodontitis by socio-economic status and inflammation in a longitudinal population-based study". Journal of Clinical Periodontology 40.3 (2013): 203-211.

\section{Assets from publication with us}

- Prompt Acknowledgement after receiving the article

- Thorough Double blinded peer review

- Rapid Publication

- Issue of Publication Certificate

- High visibility of your Published work

Website: www.actascientific.com/

Submit Article: www.actascientific.com/submission.php

Email us: editor@actascientific.com

Contact us: +919182824667

Citation: Varsha Kanjani., et al. "Evaluating the Association of Chronic Periodontitis with Quality and Duration of Sleep in North Indian Population Using Pittsburgh Sleep Quality Index - A Case Control Study". Acta Scientific Dental Sciences 6.2 (2022): 82-87. 